Revue scientifique sur la conception et l'aménagement de l'espace

19 | 2018

L'imagerie du paysage

\title{
L'imagerie irlandaise, du stéréotype au simulacre
}

Irish Imagery, From Stereotype to Imitation

\section{Corinne Feïss-Jehel et Pierre-Jérôme Jehel}

\section{OpenEdition}

\section{Journals}

Édition électronique

URL : http://journals.openedition.org/paysage/350

DOI : $10.4000 /$ paysage.350

ISSN : 1969-6124

\section{Éditeur :}

École nationale supérieure du paysage de Versailles-Marseille, Institut national des sciences appliquées Centre Val de Loire - École de la nature et du paysage, École nationale supérieure d'architecture et de paysage de Bordeaux, École nationale supérieure d'architecture et de paysage de Lille, Agrocampus Angers

\section{Référence électronique}

Corinne Feïss-Jehel et Pierre-Jérôme Jehel, «L'imagerie irlandaise, du stéréotype au simulacre », Projets de paysage [En ligne], 19 | 2018, mis en ligne le 01 décembre 2018, consulté le 28 novembre 2019. URL : http://journals.openedition.org/paysage/350; DOI : 10.4000/paysage.350

Ce document a été généré automatiquement le 28 novembre 2019.

Projets de paysage 


\section{L'imagerie irlandaise, du stéréotype au simulacre}

Irish Imagery, From Stereotype to Imitation

Corinne Feïss-Jehel et Pierre-Jérôme Jehel

1 Du livre de Kells aux clichés de la verte Irlande, il s'est développé une intense production d'images qui a façonné le visage de l'Irlande. Les paysages et leurs imageries y occupent une place privilégiée. Le secteur touristique irlandais a décliné ces " paysages à voir ", en reprenant et organisant ces images attendues. Nous abordons ici ces représentations de l'île d'Irlande, d'une part, avec un corpus d'images publicitaires, d'autre part, à travers une série de photographies personnelles qui portent un regard sur ces représentations et leurs conséquences in situ. En ce sens, il est clairement assumé que l'image photographique n'a rien d'un médium objectif comme ses apparences pourraient le laisser croire. La mise en regard d'images publicitaires avec celles d'un photographe qui fonctionnent donc sur deux registres photographiques différents permet d'appuyer une analyse de cette imagerie, l'un proposant sa perception, dont le propre est d'avoir un point de vue. Le registre photographique est ici différent - et assumé comme tel - de celui utilisé par les communicants, de même que le texte relève d'un discours écrit différemment de celui mobilisé pour et par les publicitaires. L'image permet nonobstant de souligner une distance aux paysages qui pose question. Ainsi les photographies ici présentées sont soit des éléments analysés qui révèlent un discours sur les représentations du pays par leur choix esthétique, leur cadrage, leur contexte de présentation, soit des propositions de clarification ou des éléments d'analyse de cette imagerie.

2 L'attrait pour l'Irlande repose essentiellement sur ses paysages naturels. Ce sont d'abord le pittoresque et le sauvage qui sont valorisés. Les visions de "terres de contraste » forment autant de clichés ${ }^{1}$ soutenus par les discours publicitaires. Les représentations de l'île nous parviennent avant même d'y être allés. Les photographies promotionnelles préfigurent l'image de l'Irlande, puisant dans des stéréotypes que le voyageur est incité à retrouver, puis dans lesquels il va devoir évoluer. Le voyageur qui entreprend la traversée compte bien alors retrouver les images promises. C'est la force 
du stéréotype que de «faciliter la communication » (Mannoni, 1998, p. 25) en donnant à l'Irlande une image identifiable et reconnaissable par tous.

3 Mais une fois sur place le paysage va nécessiter un ajustement. Le passage du "pays » au «paysage » par une forme "d'artialisation » conceptuelle ou in situ (Roger, 1997) gravit encore un échelon quand il devient "paysage à voir ». Le paysage est indiqué (figure 1), voire délimité (figure 7), pour entrer dans le programme organisé d'une valorisation efficace. L'imagier fournit alors les éléments d'un décor où le visiteur pourra évoluer de manière rassurante. Le paysage, naturel ou urbain, peut ainsi aller jusqu'à faire l'objet d'une remise en forme complète, plongeant le voyageur dans une expérience, démultipliant le voyage dans le paysage par une plongée temporelle ou imaginaire, atteignant ainsi un effet de fiction qui avoisine la simulation en reprenant les mécanismes d'un spectacle d'attractions.

\section{L'Irlande : des stéréotypes à un imagier entretenu}

4 Le paysage est perçu comme une entité : paysage de bord de mer, de tourbière, de montagne. Il apparaît, instantanément, associé à une lecture culturelle. En Irlande, ce sont les espaces naturels auxquels des images sont immédiatement associées qui attirent d'abord le voyageur : la côte est escarpée, les vallées sont vertes et les moutons y pâturent, les tourbières émergent de la brume, les pubs aux portes peintes nous attendent (Goeldner-Gianella et Feiss-Jehel, 2013, p. 62-76). Les images touristiques fonctionnent en effet d'abord sur le ressort de leurs stéréotypes (Luginbühl, 2012). Le Fáilte Ireland, l'autorité nationale du développement touristique, a investi 10 millions d'euros en 2014 dans le Wild Atlantic Way. Les photographies et clips en vidéo ${ }^{2}$, pour la promotion de cette opération, s'alimentent de clichés et les renforcent par retour. Le parcours du Wild Atlantic Way suit $2500 \mathrm{~km}$ de route le long de la côte, balisé de plus de 3850 panneaux, signes, blocs d'informations (figure 1) et fait halte où il faut, là où le stéréotype s'incarne dans le territoire. L'implantation des signalisations (figure 1) est imposante, massive et inscrit dans le paysage des structures décalées, en contresens, eu égard à l'état sauvage qu'elles tendent à nous montrer. La répétition des installations conduit à une uniformisation qui s'inscrit en opposition par rapport à la singularité, à l'originalité, qui est avancée. Par l'imposante signalétique, le visuel est coupé, le regard est interrompu, pour paradoxalement indiquer au voyageur de porter sa vue sur le paysage. 
Figure 1. Wild Atlantic Way

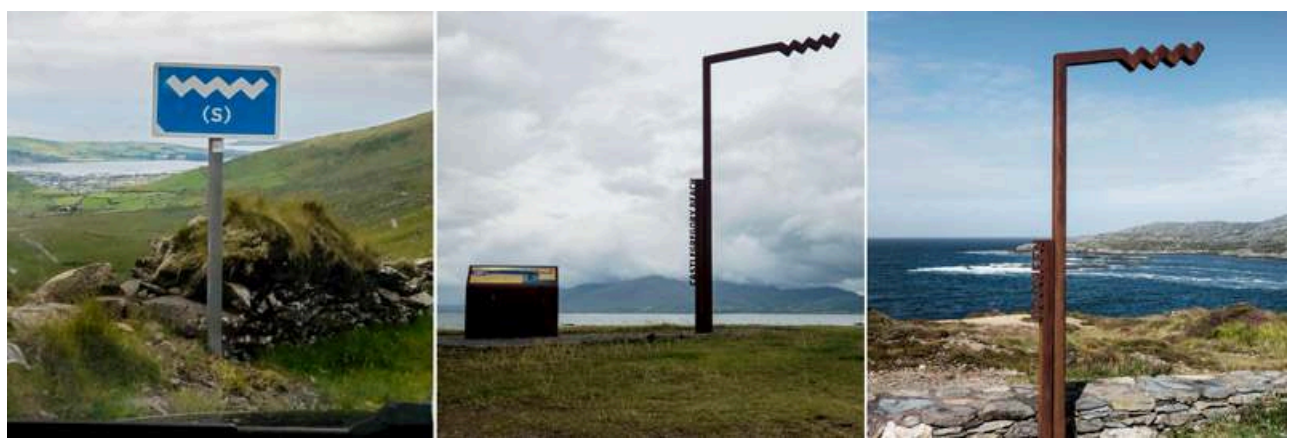

Le paysage à voir et à parcourir est signalé par un élément imposant visant à le faire appartenir à un ensemble continu considéré comme caractéristique du pays.

Photos Pierre-Jérôme Jehel, 2015, 2017, 2018.

5 Quelques sites emblématiques (plateau du Burren, Chaussée des Géants, promontoires du nord, côtes du Connemara et du Kerry par exemple) sont les fleurons de ces représentations promotionnelles. Les images sont alors accompagnées d'un apparat sémantique qui tend à augmenter leur effet. Les falaises de Moher "plongent à la verticale dans les tréfonds de l'océan déchaîné3. » Le plateau du Burren est un « désert de pierre, paysage presque effrayant ${ }^{4} . »$ (Figure 2). Concernant les falaises de la Chaussée des Géants, il s'agit d'« admirer la beauté sauvage à l'état pur ${ }^{5}$.» (Figure 3.)

Figure 2. Plateau de Burren

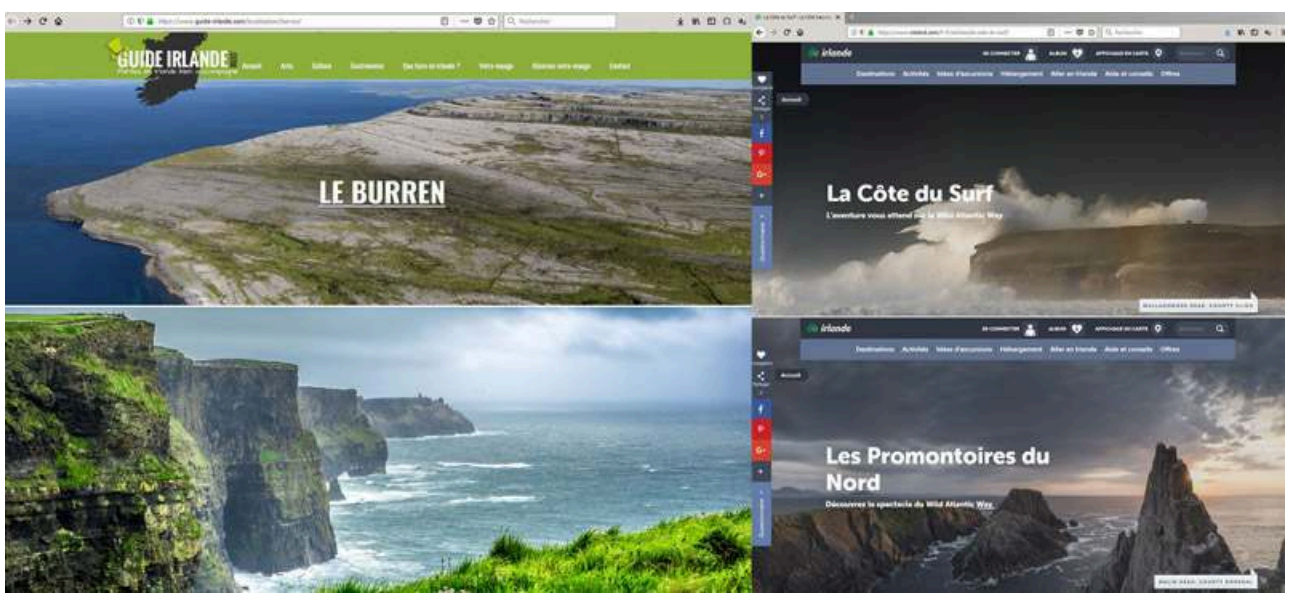

Pages extraites des sites https://www.guide-irlande.com/localisation/burren/ et https:// www.ireland.com/fr-fr/articles/wild-atlantic-way/, consultés en juin 2018.

Figure 3. " La Chaussée des Géants »

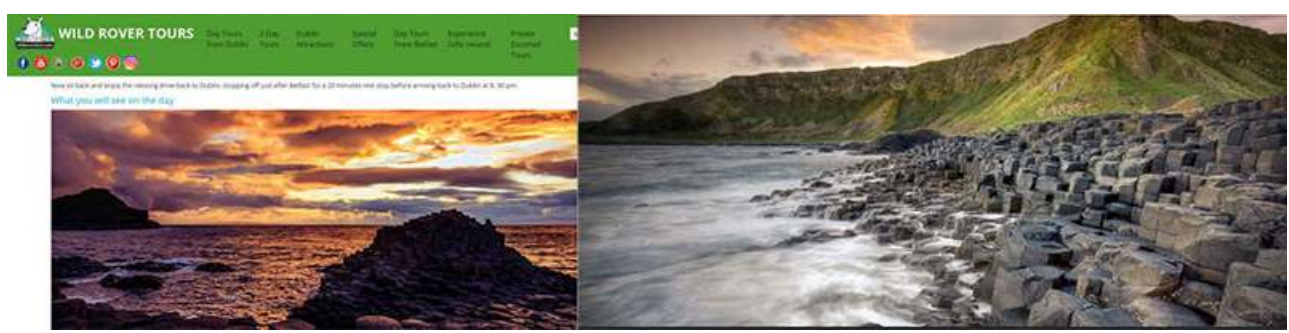

Sur le site https://wildrovertours.com, consulté juin 2018. 
6 Le paysage est ici façonné par et pour une intention touristique. Les paysages sauvages sont une forme de mythe entretenu, une nostalgie où les images produisent des valeurs iconiques. L'île rêvée est avant tout inhabitée, sauvage, un souvenir du paradis perdu (figure 4).

Figure 4.

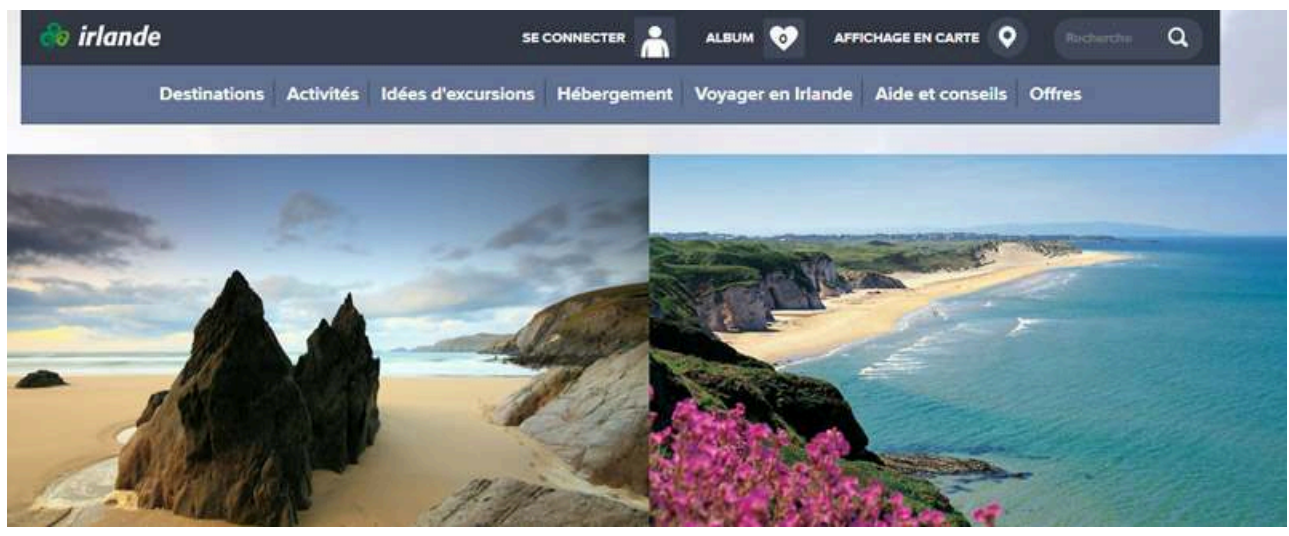

Les plages sont désertes, pas un promeneur ne vient perturber le champ.

Voir https://www.ireland.com, consulté juin 2018.

Pour atteindre l'effet désiré, les images sont artificiellement fabriquées comme dans le clip du Wild Atlantic Way et renforcées, dans leur catégorie de clichés : utilisation de filtres d'accentuation, effet de netteté, saturation débordante. Le ciel est d'un bleu idéal ou chargé de nuages menaçants. La mise en scène est complétée par une mise en lumière efficace : éclairage contrasté, percées du soleil, reflets chatoyants de la mer. Le cadrage joue un rôle essentiel, il évite les «perturbations » : fils électriques, présences humaines, voitures. La photographie promotionnelle est un artifice, une mise en scène où le monde devient celui que l'on veut. Elle définit un monde du désir contrôlable, un spectacle, au sens où « le monde réel se change en simples images, les simples images deviennent [...] les motivations efficientes d'un comportement hypnotique » (Debord, 1967, p. 23).

Le paysage ainsi fixé dans sa représentation paraît immuable. Ces photographies créent un espace hors du temps où chacun peut y glisser l'image de soi dans un cadre social et faire partie du spectacle. Ce monde du point de vue n'est finalement qu'un leurre, car le voyageur va pour voir et photographier une forme d'image-témoin qu'il rediffusera.

Ce paysage idéalisé rappelle la veduta des peintres de la Renaissance où l'environnement perçu à l'extérieur n'était pas l'image fidèle d'un paysage identifiable, mais une vue composée à partir de plusieurs paysages ${ }^{6}$. Mais ici, il ne s'agit pas de reconstituer un espace rêvé ou fantasmé, telle une métaphore d'un pays imaginaire ou inconnu, mais de se conformer à l'image qui a été imposée. Les images de paysages diffusées par l'office du tourisme irlandais sont une mise en scène d'un territoire dans lequel le voyageur aime se retrouver. L'Irlande ainsi photographiée est d'ailleurs à encadrer et à exposer chez soi telle quelle. Si le ciel bleu n'était pas au rendez-vous, qu'importe ! Il a déjà été pris en photo pour nous et nous pouvons acquérir cette image, comme elle aurait dû être. Le site du Wild Atlantic Way nous propose de l'envoyer chez nous (figure 5). Différents formats, supports et encadrements sont en ligne pour les adapter à notre intérieur. 
Figure 5.
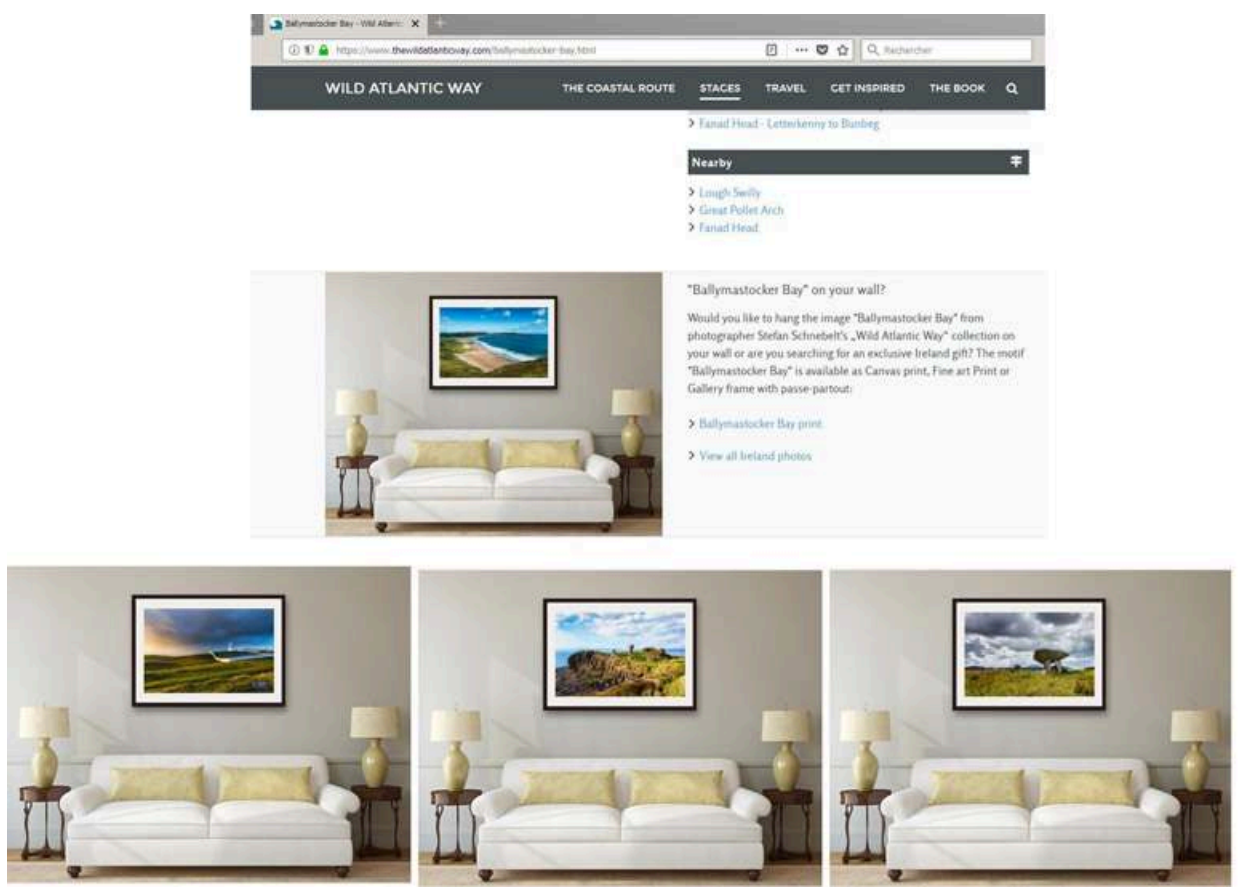

Les paysages sont déjà prêts, photographiés.

Voir https://www.thewildatlanticway.com, consulté juin 2018.

10 Mais paradoxalement, les intérieurs où viennent prendre place ces images sont également étrangement vides et impersonnels. Le paysage dont nous serons ou avons été le héros est bien là et nous le partagerons avec nos invités. Mais il est sans nous. On pense d'ordinaire l'image photographique comme le témoin qui doit authentifier la présence du touriste à un moment donné dans un paysage précis. Dans ce cas, le voyage n'apparaît pas comme une affaire personnelle mais configure une image pour les autres. Nous entrons dans une représentation qui est essentiellement sociale. Le paysage est alors avant tout celui des sentiments complexes de la représentation. Nuances, situations et émotions s'effacent au profit d'un répertoire social codifié en stéréotype. La photographie n'ouvre pas sur une expérience particulière mais sur une recherche des paysages "typiquement irlandais». S'il y a déterritorialisation par l'homogénéisation des clichés et de leur cadrage, il y a identification par les lieux retenus et leur mode d'artificialisation qui symbolisent l'Irlande sauvage. L'accentuation de la densité des ombres, le renforcement des contrastes dans des cieux nuageux accompagnent cet ancrage dans la représentation stéréotypée (figure 5).

En appuyant sur le déclencheur, nous passons acteur et participons à la communauté. Les fenêtres, les windows numériques captent, enregistrent, transmettent et se rechargent d'images collectées. Les images de nos écrans sont immédiatement connectées aux réseaux sociaux et pourtant, paradoxalement, elles peuvent faire écran à la réalité du voyage. Avec le selfy, le paysage quitte le devant de la scène, il devient le décor d'une scène de vie. Mais le «je du selfy » doit toujours apparaître dans une Irlande reconnue par tous. Il ne doit pas tant avoir vu qu'être vu. La photographie redevient preuve, témoin. Les photos « documentent la vie, participent au jeu de l'autoprésentation et servent à des fins référentielles » (Gunthert, 2014). L'ère des selfies est celle où l'image se confond avec un désir d'afficher une existence. L'acte 
photographique individuel conforte et amplifie la représentation sociale choisie. L'Irlande éternisée est perçue et reconnue dans sa doxa visuelle. Les paysages touristiques s'approchent du mythe au sens où Roland Barthes écrivait «le mythe est une parole » (Barthes, 1970) : il est un système de communication, un message lié à une certaine société à un moment précis de son histoire. Et si «le mot paysage désigne l'espace adéquat d'une rêverie, productrice d'idées, créatrice d'envies" (Ehrmann, 2015), la question se pose de savoir si le rêve, reflet d'une interaction intime et personnelle avec cet espace, est encore permis dans un paysage de mise en scène.

\section{De l'imagier à la mise en décor}

Pour parfaire l'organisation des regards sur le terrain, des aménagements paysagers constituent une sorte de muséographie des territoires. La construction des paysages types constitue une métamorphose des lieux. Le territoire devient un catalogue de paysages : "On a affaire à une métastase généralisée, à un clonage du monde et de notre univers mental... Nous devenons non plus les spectateurs aliénés et passifs mais les figurants interactifs, les gentils figurants lyophilisés de cet immense reality show» (Baudrillard, 1996).

L'idée d'un monde rural perdu, de terres résistant à la mondialisation, de landes face à l'océan constitue un territoire de l'imaginaire. Ces bastions sont ancrés sur des références picturales romantiques. Les paysages de mer sont teintés par les préimpressionnistes et les impressionnistes (Vague de Courbet; Étretat, soleil couchant de Monet). Les visiteurs de paysage parcourent les sites comme des salles de musée, appareils photographiques en bandoulière et audioguide à l'oreille (figures 6,7,8). Le stéréotype formalise alors spatialement un paysage non plus attaché à une réalité naturelle locale ou historique, mais comme le décor visuel d'un show distrayant. Cette formulation des paysages nous conduit à une mise en conformité entre paysage rêvé et paysage vécu. Le « désir du rivage » (Corbin, 1990) qui émergea au tournant du XVIII ${ }^{e}$ siècle se nourrissait d'émotions nouvelles mais la question se pose de savoir si à présent nos désirs n'ont pas été « amputés, formatés, appauvris par l'omniprésence des propagandes commerciales» (Gorz, 2005, p. 115).

Figure 6. Plateau du Burren
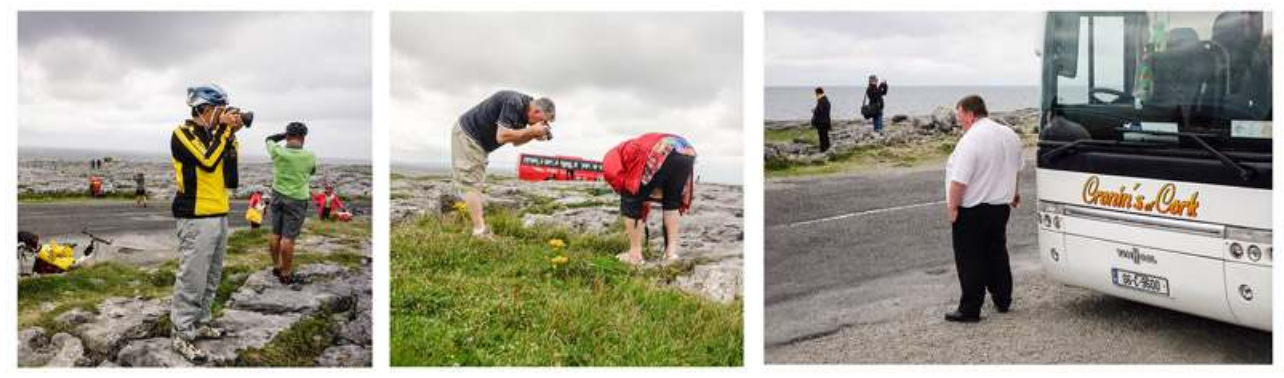

À travers les calcaires du Parc national du Burren, les voyageurs s'arrêtent aux lieux indiqués pour profiter un temps de ce paysage et de sa flore sauvages. Une certaine frénésie semble interdire la promenade au profit d'un parcours à rythme soutenu d'un site à l'autre.

Photos Pierre-Jérôme Jehel, 2013. 
Figure 7. Cliff of Moher
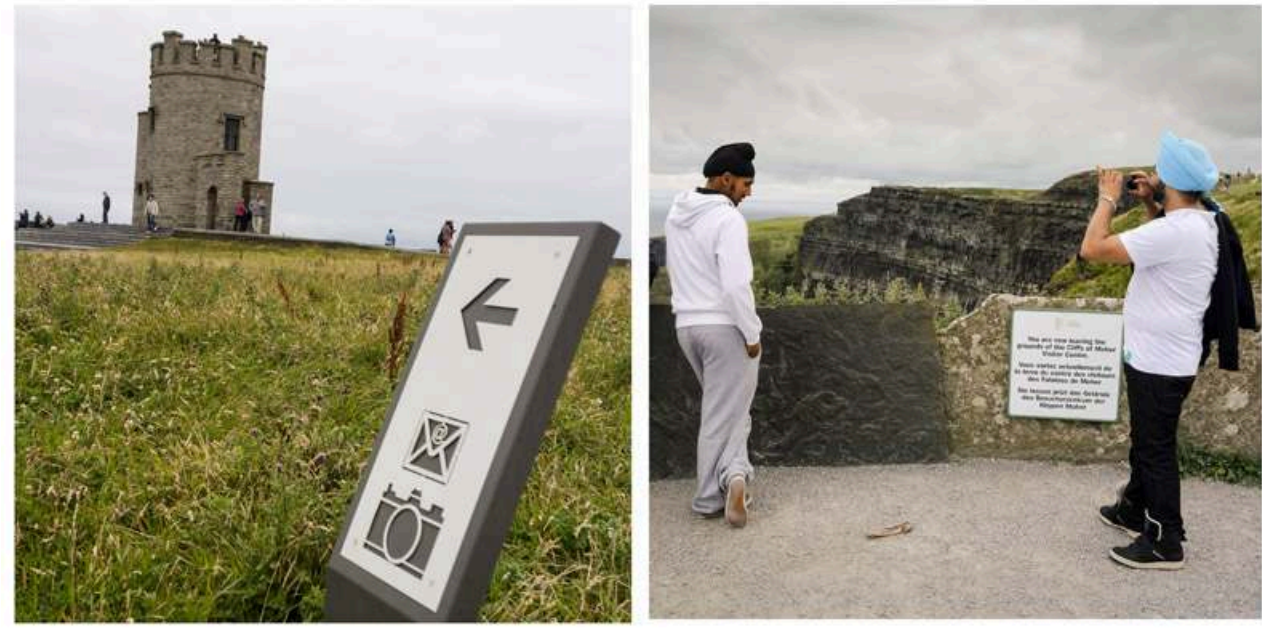

Le site est balisé de panneaux indiquant les limites du Visitor center. À l'intérieur, les fléchages indiquent les points spectaculaires à photographier et l'accès au réseau pour partager immédiatement ces clichés.

Photos Pierre-Jérôme Jehel, 2013.

Figure 8. Giant's Causeway, 2013

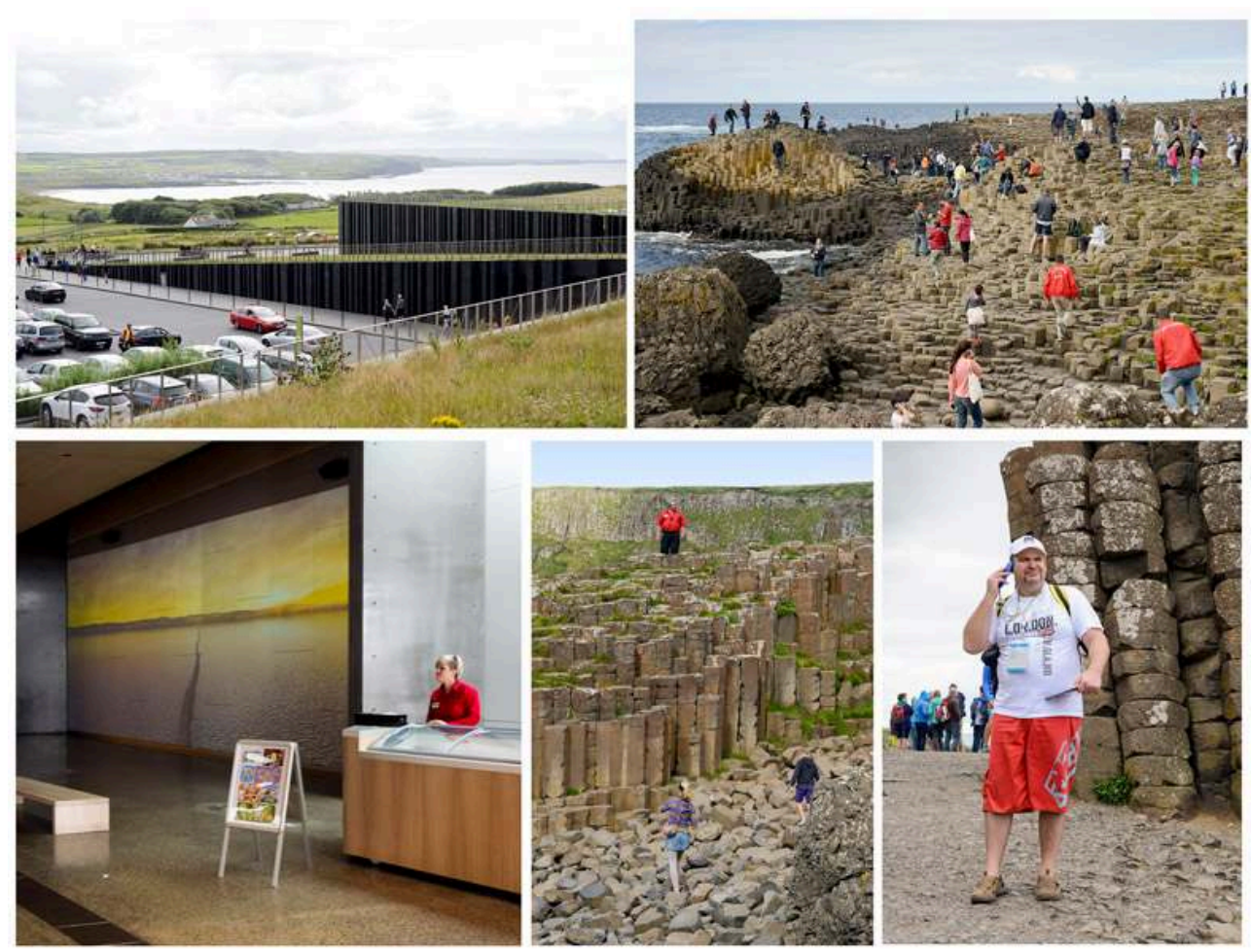

Le très célèbre site de la Chaussée des Géants connaît un nouveau centre d'information depuis 2012 doté de deux parkings qui marquent l'arrivée sur les lieux. On accède au site lui-même après les différentes animations interactives. Sous la surveillance des gardiens qui délimitent la zone, la foule de promeneurs se disperse dans le paysage, un audioguide est mis à disposition pour indiquer encore plus précisément les éléments à ne pas manquer. 


\section{De la simulation aux simulacres} l'agriculture intensive (figure 9). Nous allons nous aussi « faire à la main ». « Give visitors the opportunity to experience living Irish culture ${ }^{10} »$ nous lance le guide du site touristique. La nostalgie que nous avions d'un certain passé nous a rattrapés et nous est proposée dans un monde parfait. Les photographies du site nous enjoignent de rejoindre la famille. Le cadrage subjectif plongeant sur les pieds nus sur la terre nous implique, et nous incite directement à venir vivre cette expérience à remonter le temps et à retrouver des sensations perdues. Nous touchons le pain à l'ancienne, la tourbe, même la boue. L'expérience est lissée, elle a transcendé le modèle en éliminant les troubles, les vicissitudes, les perturbateurs de la vie quotidienne. Ces lieux s'adossent sur une résurrection des ustensiles anciens, d'un vécu idéalisé et heureux que nous pouvons partager. Nous repartirons avec un morceau de passé que nous nous approprierons comme souvenir. Une expérience passée, vécue dans le présent, dans une production de « réel» référencée et entretenue. Nous pouvons choisir en toute quiétude nos futurs souvenirs : époque, thème... à nous de les faire vivre en y transposant notre moi. Le lieu est le support d'un album-souvenir où les images représentent le discours dont on veut se souvenir. Pour John B. Jackson, à la fin du XIXe siècle, de nouveaux monuments sont apparus, qui mettaient en avant non plus de grandes figures politiques ou morales, mais des anonymes. On visite de vieilles chaumières, des fermes, et même des chaises. «Ces monuments célèbrent une autre espèce de passé, pas celui que décrivent les livres d'histoire, mais un passé vernaculaire, une espèce d'âge d'or sans noms ni dates, un simple sens de la façon dont les choses étaient autrefois, l'histoire comme chronique du quotidien. » (Jackson, 2005, p. 146 sq.) Les jardins enfouis, les boutiques désuètes et les vieux métiers pittoresques d'autrefois témoignent d'un passé enchanteur. La mise en patrimoine des bâtiments comme de leur cadre environnemental procède autant de la protection « d'un âge d'or » du pittoresque que d'une esthétique de l'ancien.

Nous recherchons l'image du temps disparu, une formulation visuelle du temps du souvenir, du temps du bonheur. Et si la mémoire, comme le suggère Aristote, « est la 
présence dans l'esprit de l'image, comme copie de l'objet dont elle est l'image », les objets quotidiens muséifiés et encore en mouvement sont là pour nous rappeler la "réalité » et ancrer les souvenirs. Mais l'image du souvenir perd sa liberté, son interprétation par rapport à une réalité technique et doit correspondre à ce qui nous est expliqué. Il se forme alors un chevauchement entre mémoire et image nouvelle du temps passé.

Figure 9. Sites d'informations sur The Causey Farm

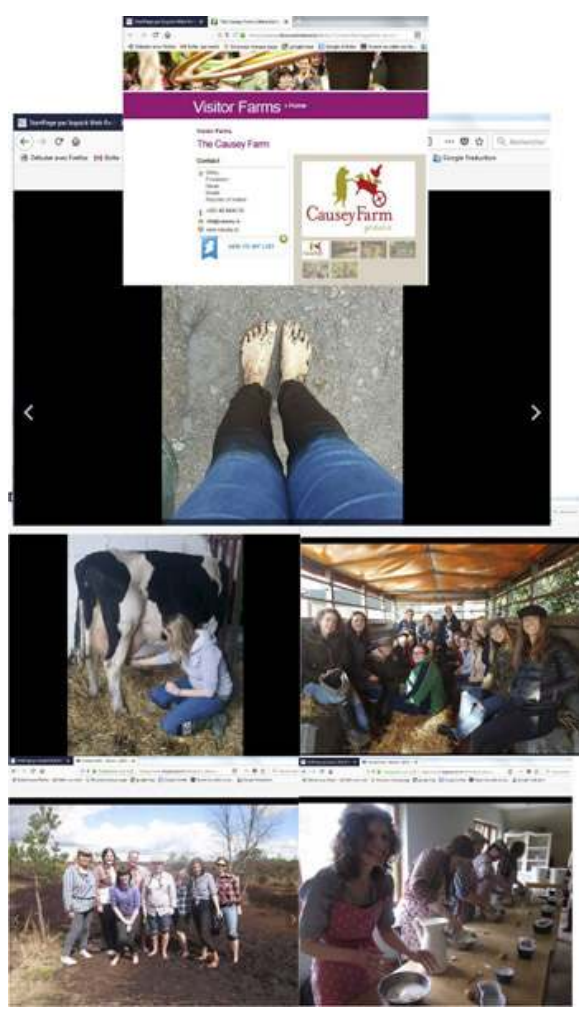

Les expériences proposées sont identiques pour chaque session et réitérables. Elles sont transformées en une dimension réelle de notre vie et, de façon plus critique, nous empêchent d'imaginer.

Avec Navan Fort, l'expérience est du même ordre mais en exploitant la veine historique. 
Figure 10. Sites d'informations sur Navan Fort

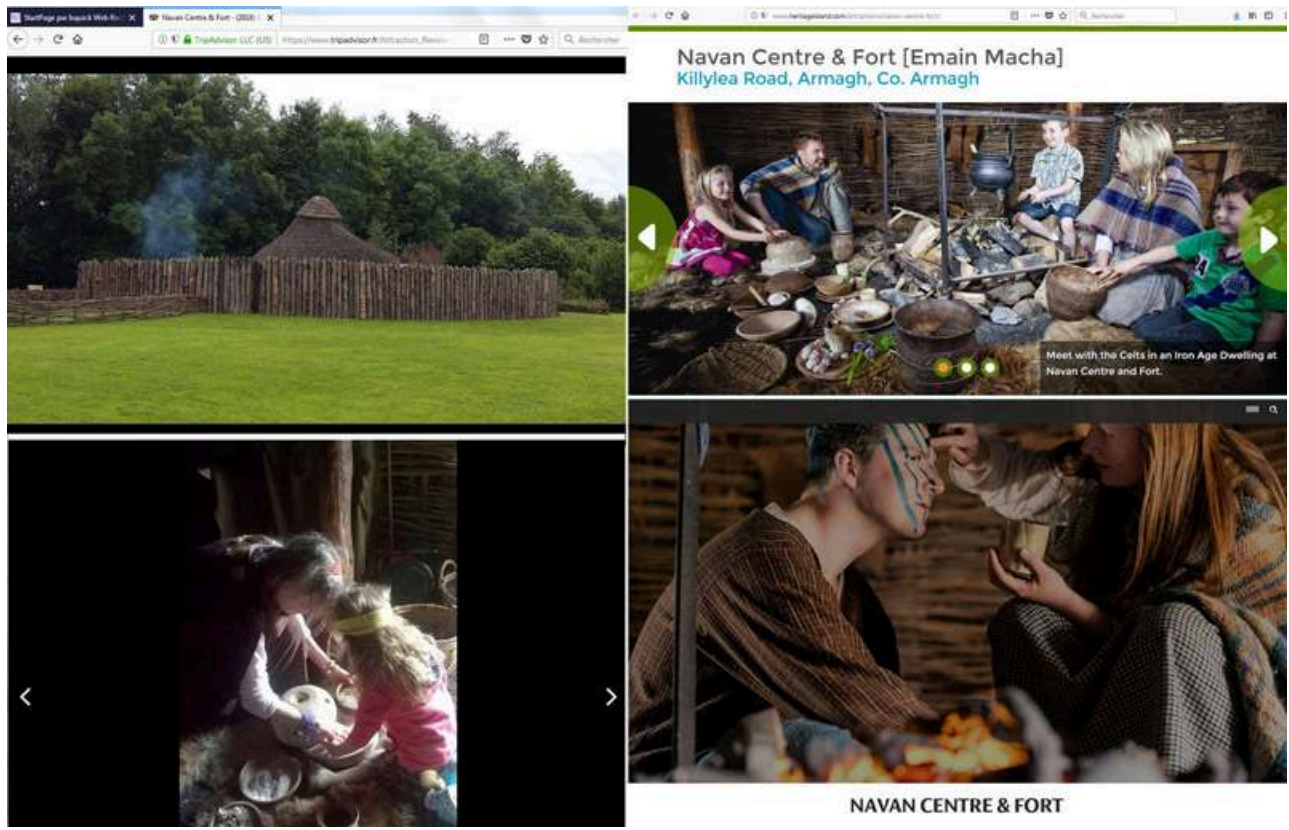

Les aménagements et recompositions sont chirurgicalement historiques car la simulation mise en place fonctionne et ne vaut que par sa garantie de «réel » (figure 10). Le temps de la civilisation préchrétienne de l'âge de fer, le cycle de l'Ulster s'ouvre à nous. Cúchulainn et le monde mythique nous sont restitués et nous donnent une lecture triée d'un passé sélectionné pour son histoire, ses personnages. L'image recomposée et conservée avec un déploiement d'hyperréalisme est bien plus forte que l'histoire d'origine. La fumée même sort encore du petit village par-dessus la fortification de bois (figure 10). Les images promotionnelles nous fournissent un imaginaire préenregistré afin d'anticiper le réel. Nous sommes peut-être dans une situation utopique au sens de Louis Marin (1979) :

"L'Utopie est une île [...] que l'on peut considérer comme une sorte de présent intemporel. Il s'agit d'une consommation d'expérience qui participe à ce que $\mathrm{M}$. J Wolf ${ }^{11}$ appelle "experience economy" ".

Le Titanic Center muséifie quant à lui équipages, passagers, hommes, femmes, de seconde comme de première classes, un pan de la société entière. "Un musée qui redonne vie au Titanic. » Nous pouvons vivre « au cœur du paquebot, [...] descendre le grand escalier de la salle à manger ", nous "placer devant la barre du poste de commande ${ }^{12} »$ (figure 13). Tout est beau, même le naufrage et la mort.

"Les musiciens jouant sur le pont jusqu'au dernier moment, les actes de bravoure [...] le tragique décès du capitaine Smith, affrontant les eaux face à sa barre de commandement. Bref des histoires dramatiques, chargées en émotion ${ }^{13}$. »

Nous sommes dans le déploiement d'une réalité accompagnée de son cortège d'émotions. Il existe une sorte de surenchère du " vrai », les visiteurs installés dans une nacelle font l'expérience des chantiers Harland \& Wolff où des effets spéciaux et des animations se relaient pour faire sentir, entendre la construction navale comme à l'époque puis, au travers d'une grande baie vitrée, équipée d'électrodes, qui enchaîne vue réelle et images superposées du Titanic, ils assistent au lancement du Titanic... L'objectif muséographique précise Dominique Chevalier (2014) «repose sur l'idée 
performative que les visiteurs, à l'issue de leurs expériences physiques et émotionnelles, seront de formidables agents du succès et de la réputation des lieux ».

Figure 11. Expériences immersives

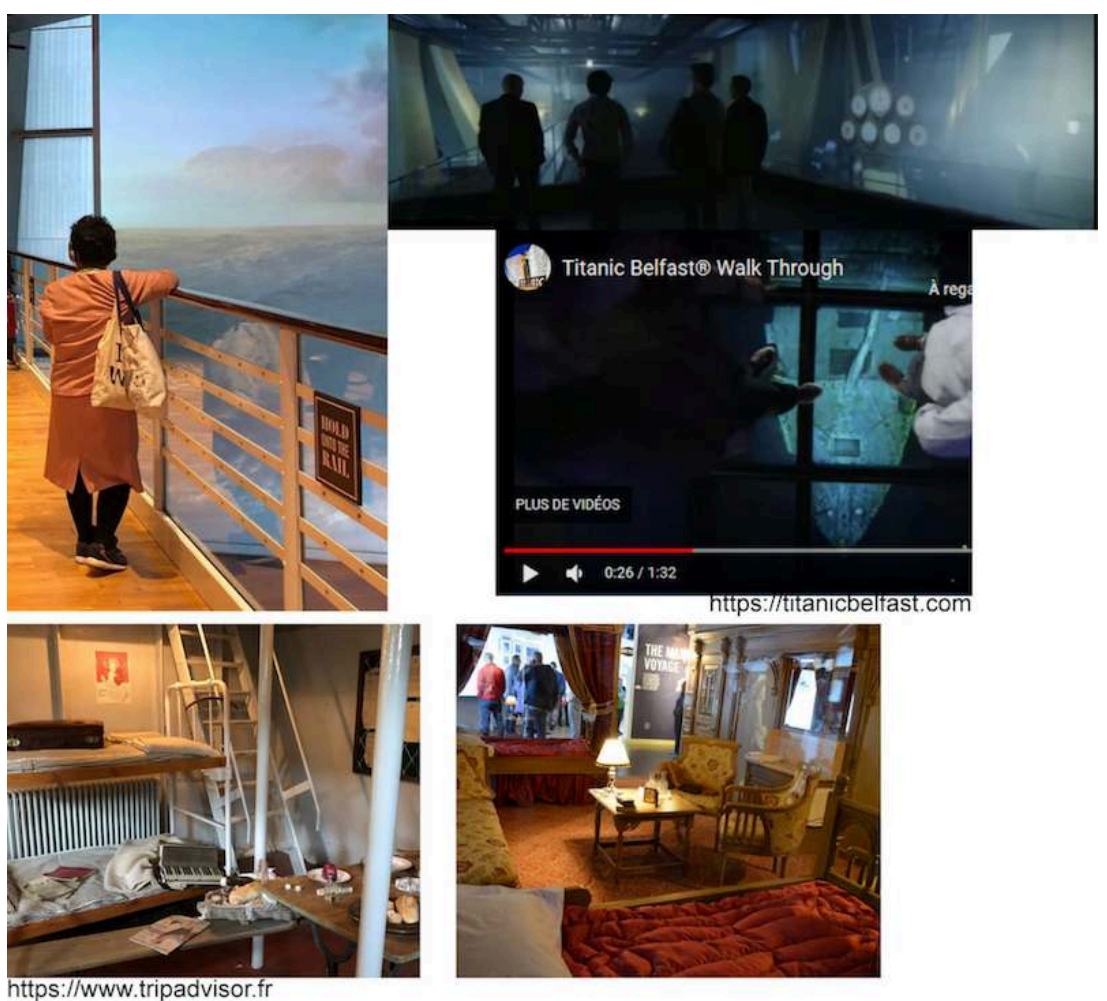

Nous pouvons nous accouder au bastingage et profiter du large, descendre vers les ponts inférieurs, vers les cabines ou surmonter l'épave depuis une plaque de verre comme si nous étions dans le sousmarin qui l'a découverte. Le pain est encore frais sur la table et il semble que l'on vienne juste de quitter la cabine.

Les fastes de la Belle Époque, le naufrage, la mort : tout est présenté. Il s'opère une rationalisation de l'événement. Un sujet aussi tragique que le Titanic peut alors devenir un terrain de divertissement culturel, ce qui tend à souligner comment «le divertissement et les lois du business du divertissement pénètrent de plus en plus [...] le monde de la culture » (Wolf, 1999, p. 28).

Les anciens docks ont été eux aussi investis d'un attrait touristique grâce à la marque Titanic. Sur une logique de parc d'attractions, le Titanic Quarter accueille les visiteurs en quête de retrouver le temps des transatlantiques (figure 12). Les chantiers navals noirs de graisse ont été repeints, les bâtiments de brique sont restaurés, la rouille devient un élément décoratif. Les espaces de travail industriel et portuaire sont devenus espaces de loisir. 

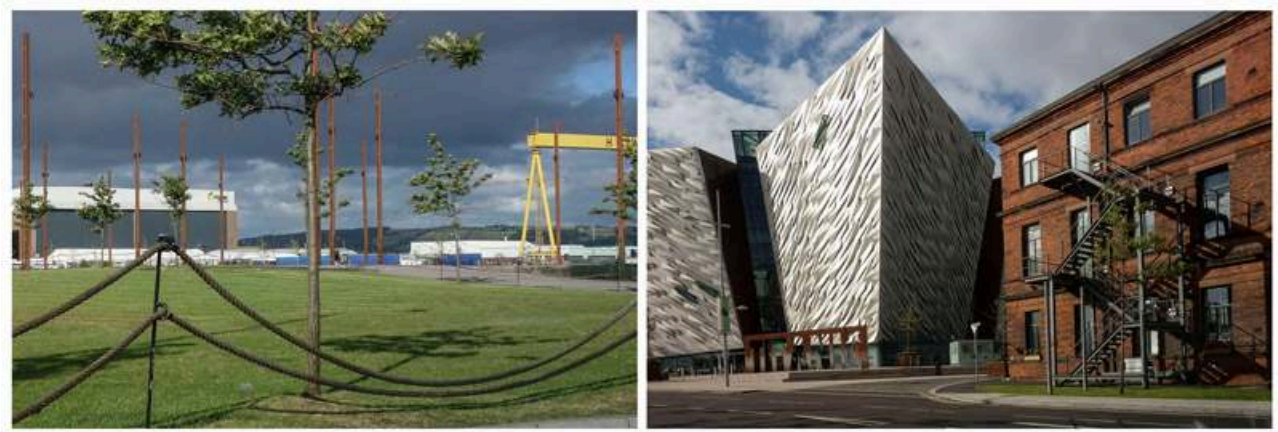

Photos Pierre-Jérôme Jehel, 2015.

Figure 13. Titanic Center
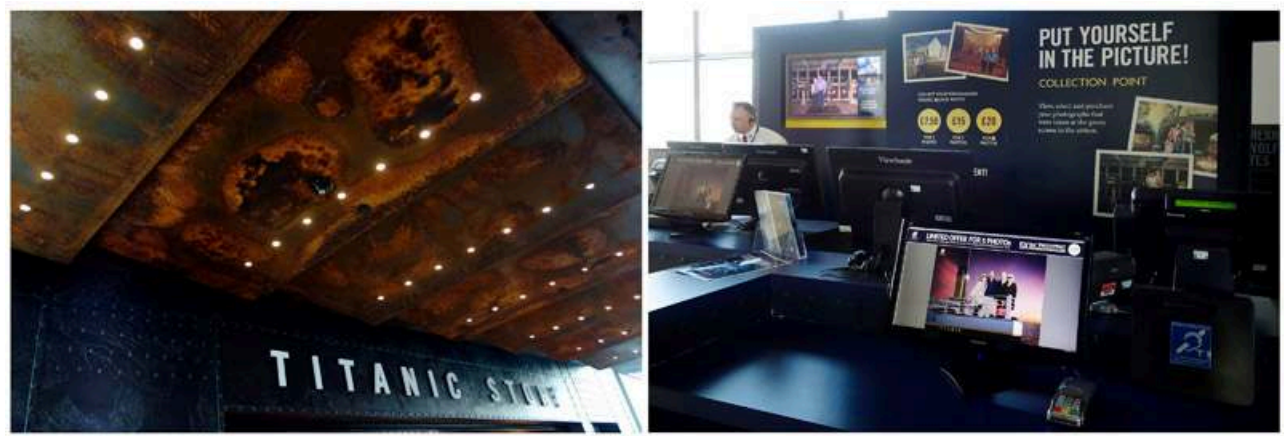

Photos Pierre-Jérôme Jehel, 2015.

Ces nouveaux espaces semblent prêts pour désormais susciter, diriger et discipliner les rêves, les désirs et les besoins des touristes. Ce ne sont pas de simples décors puisqu'ils conditionnent intégralement l'expérience, ce sont des dispositifs immersifs.

Les paysages, les quartiers, les villes ont été réinvestis pour devenir patrimoine naturel et/ou espace de loisirs. Ils doivent satisfaire à de nouveaux usages qui se traduisent par une reconfiguration $\mathrm{du}$ territoire tant d'ordre symbolique qu'économique. Les politiques de conservation sont généralement énoncées et soutenues par l'État ${ }^{14}$. Les réaménagements de quartiers comme Titanic Quarter nécessitent des investissements plus importants et se font par des fonds privés ${ }^{15}$. Le Titanic Quarter accueille aujourd'hui plus d'un million de visiteurs chaque année et représente 425 millions de livres d'investissements de la part d'une des plus importantes sociétés immobilières privées d'Irlande : Harcourt Developments. Ses actifs se répartissent sur des bureaux, des centres commerciaux, des parcs industriels, des attractions touristiques et des hôtels dans neuf pays différents. Ils énoncent ainsi leur philosophie : «Intégrer des éléments culturels et artistiques dans tous nos projets. » Les investissements dans la régénérescence des quartiers des docks ont conduit également vers une «Titanification » de l'urbanité. Philip Rea (2012) place les enjeux du développement du quartier du Titanic à l'échelle internationale. Les nouveaux quartiers se superposent à une homogénéisation culturelle et à une forme particulière de commercialisation de la culture.

28 La ré-imagerie postindustrielle passe par une configuration de significations qui est impliquée dans la perception de la ville et portée par l'image de la régénération. Belfast 
intègre la mondialisation par son Titanic Quarter fondée sur une économie culturelle créative mais «les formes spatiales» attractives des quartiers centraux ou péricentraux se sont transformées « en agrégats de fragments fortifiés » (Harvey, 2010) de plus en plus homogènes où les ouvriers ont disparu pour laisser l'espace aux touristes et aux hommes d'affaires. L'importance du Titanic de Belfast, réelle ou supposée, devient la marque du projet et valide la notion d'héritage. L'identité urbaine et l'expérience urbaine ainsi créées jouent comme un pivot pour le tourisme et l'économie de services. La ville devient spectacle et le touriste spectateur. La ville est repositionnée comme un musée à expérimenter et l'espace urbain se lit en termes d'aménités pour les nouveaux résidents.

29 Avec les murals de Belfast et de Derry, c'est l'histoire contemporaine qui devient un sujet touristique. Longtemps évitées, cachées, les contestations de rues entre protestants et catholiques sont réincorporées à une marchandisation possible. Les villes de Belfast et Derry ${ }^{16}$ ont vu glisser leurs murals d'intimidation et de revendication vers un art de ville, une forme épurée de street art à visiter (figures 15, 16 et 17).

Figure 14. Belfast

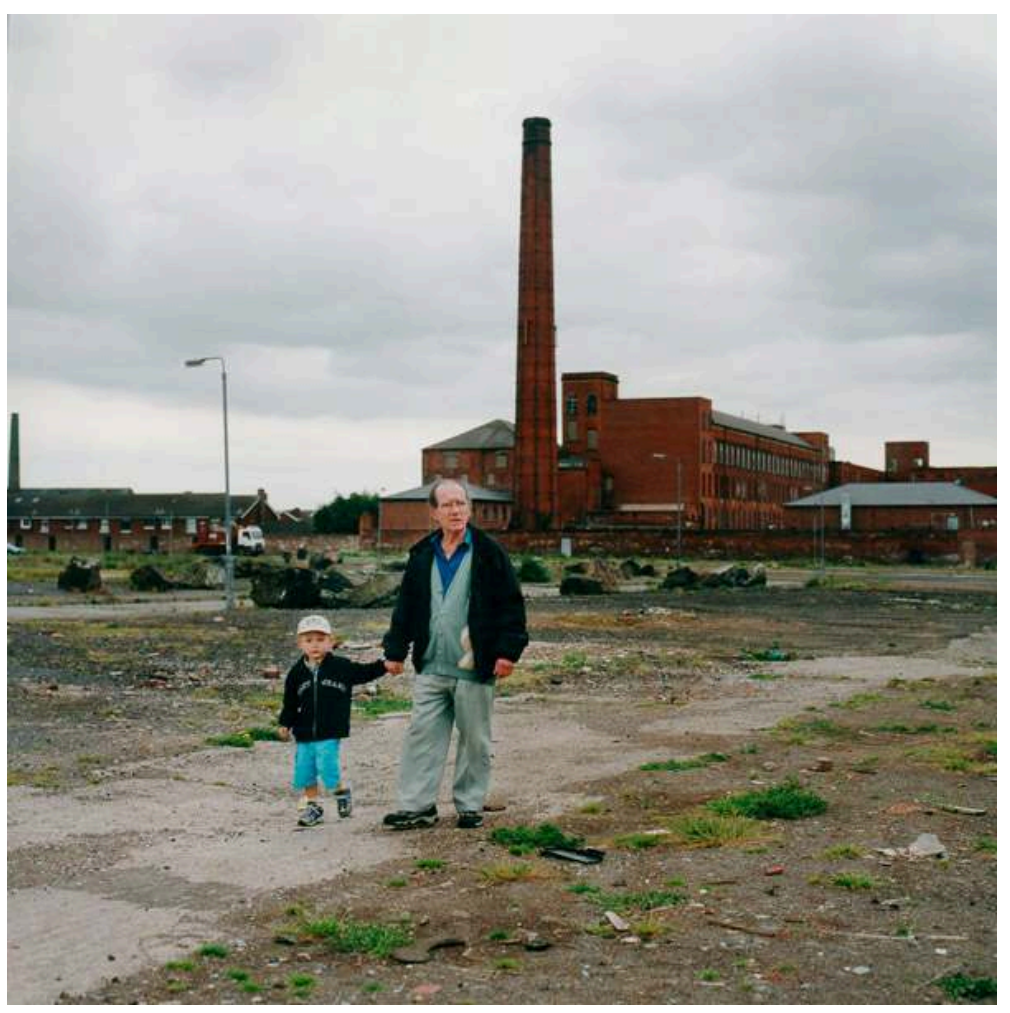

Photo Pierre-Jérôme Jehel, 2001. 
Figure 15. Belfast

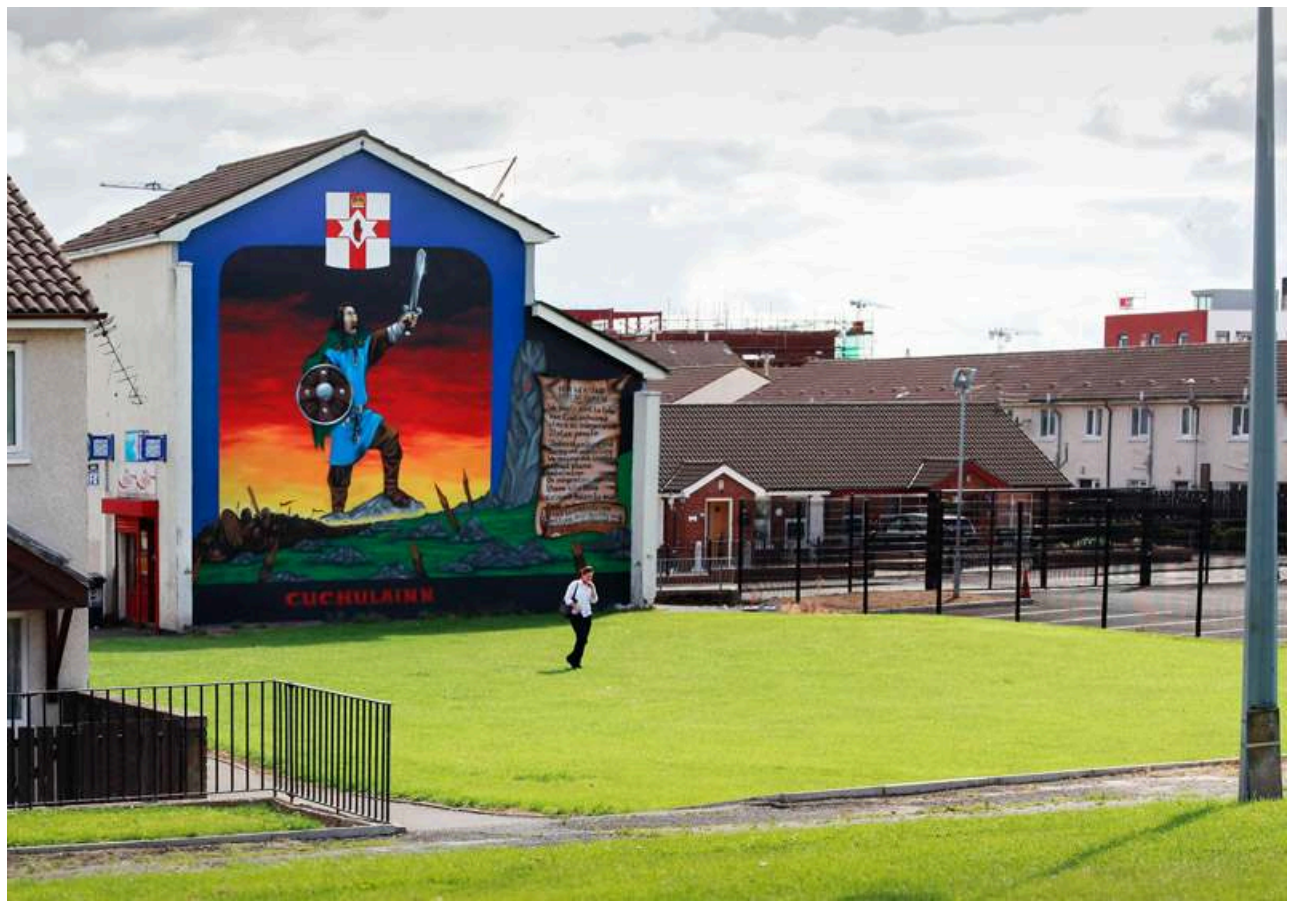

Photo Pierre-Jérôme Jehel, 2010.

Figure 16. Derry

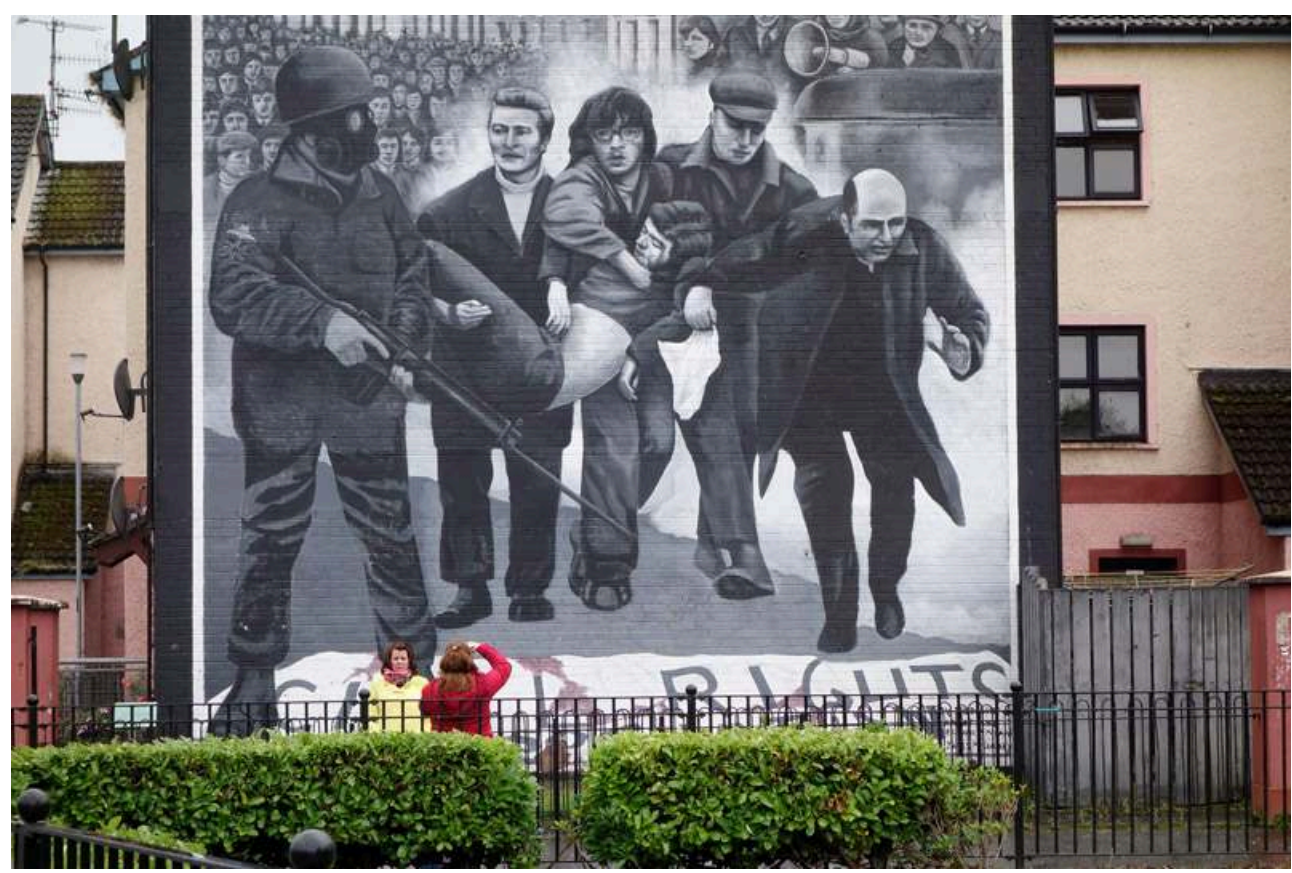

Photo Pierre-Jérôme Jehel, 2015. 

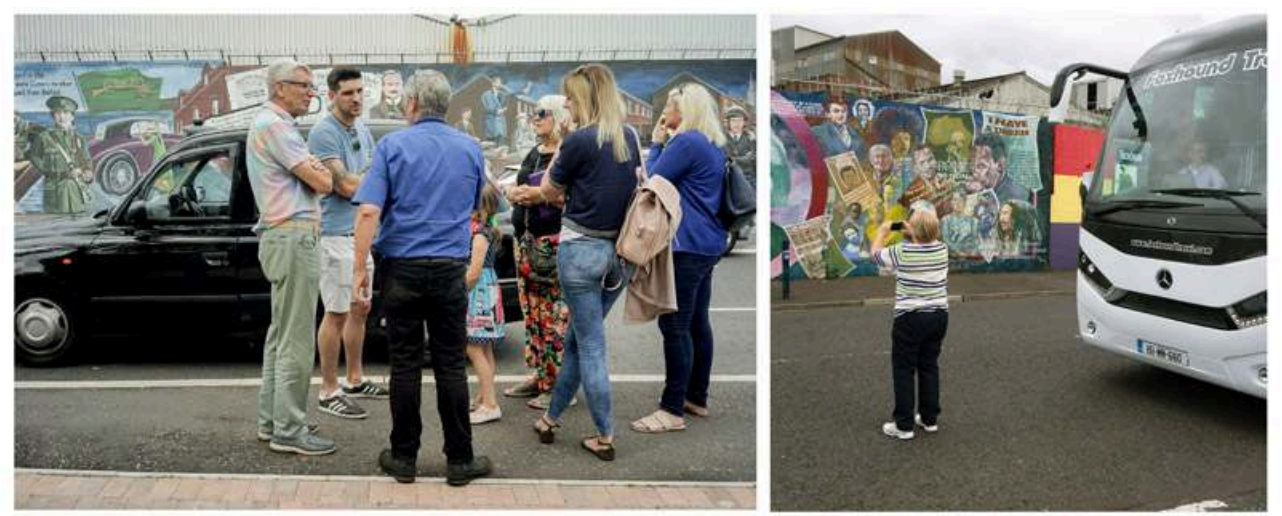

Photos Pierre-Jérôme Jehel 2017, 2015.

En 2001, les stigmates des émeutes étaient toujours bien visibles dans les quartiers de Belfast (figure 14) puis les zones ont été nettoyées et rendues présentables. Les peintures murales et la pelouse apparaissent soigneusement entretenues. Quelques années plus tard, les touristes et leurs appareils photo parcourent les walls et les quartiers qui ont été réaménagés (figure 15). À Derry, des parcours similaires sont tout autant objets de photographies (figure 16), devant une fresque à la mémoire du Bloody Sunday. Florine Ballif (2015) note que les guides des éditeurs internationaux les présentent comme étant «des lieux incontournables» et que les médias les citent " comme les premières attractions touristiques au Royaume-Uni ${ }^{17}$ ".

31 La transformation de ces espaces mutilés en zones à valeur économique était difficile à concevoir. Les fresques murales de Belfast, souvenir brûlant de la guerre civile, ont été repeintes et font même l'objet de panneaux explicatifs. Des circuits (figure 17) sont proposés et s'organisent sur le développement du dark tourism (Lennon, Foley, 2000). Les terrains vagues, les rues interrompues entre le quartier autour de Shankill Road des unionistes, d'une part, et celui autour de Falls Road catholique, d'autre part, parsemés de pavés et de projectiles divers, ont été engazonnés (figure 14) et même si ce vert si irlandais correspond à l'utilisation régulière de nitrate, il offre un aspect plus conforme. Les ruelles mitoyennes, étranges couloirs aux murs rehaussés de briques puis de barbelés, ont été rénovées. L'histoire et ses fractures ont été réinvesties. Le voyageur est invité à visiter les places traumatisées du conflit historique et un canevas des pignons repeints tisse la promenade. Bien lisses, sans impact, ces fresques murales restaurées in situ balisent le chemin des randonnées urbaines. Le détournement joue ici à contresens. Il n'est pas provocation, intimidation ou marquage de territoire comme les fresques d'origine, mais réintégration et réappropriation par l'économie. Les portes blindées des « peacewalls» qui fermaient les quartiers ont été conservées et, comme les murs d'enceinte de Philippe-Auguste à Paris, font partie de la visite. L'histoire nous est contée par un guide expert :

«Our Belfast Murals Taxi Tour is guaranteed to educate, enlighten and entertain ${ }^{18}$."

La possession d'un patrimoine est une marque de la modernité et « la vie devient patrimoine avant même qu'elle ait une chance d'être vécue » (Kirshenblatt-Gimblett, 2006, p. 161-202). La question indirecte qui se joue est celle du passé. Manquerions-nous finalement de passé ? Cette régénération urbaine renforce une ségrégation urbaine, les 
quartiers réinvestis et centraux sont destinés aux touristes, à la classe d'affaires et les classes ouvrières sont déplacées vers les espaces péricentraux.

Nous avons glissé vers une commodification ${ }^{19}$ des paysages (Appadurai, 2005). L'homogénéisation est à la fois visuelle et culturelle. Elle conduit à une commercialisation de tous les espaces, et ce d'abord par l'image.

\section{Conclusion}

34 Le monde photographié reflète les mutations de la société au sens où les paysages deviennent décors. Les stéréotypes constituent un imagier qui met en avant une muséographie des territoires où il faut voir, faire, apprendre et comprendre. Les paysages-images retenus constituent une forme de conservatoire du paysage. Nous franchissons encore un seuil quand nous entrons dans des mondes clos où «le réel luimême devient un parc d'attractions" (Baudrillard, 1996). La simulation de la réalité devient un objet de consommation visuelle.

Cette consommation agit comme un critère majeur d'identification individuelle. Sur Facebook, Instagram, la personne se décrit par une identification aux livres, à la musique, aux séries, aux lieux de vacances qu'elle consomme mais et surtout par les images qu'elle montre d'elle-même pour s'inscrire dans un référentiel. Guy Debord, dans La Société du spectacle (1967) écrit que :

«Tout le temps consommable de la société moderne en vient à être traité en matière première de nouveaux produits diversifiés qui s'imposent sur le marché comme emplois du temps socialement organisés. »

La consommation d'espace devient un produit au même titre qu'une série télévisée de Netflix. Nous en sommes aux prémices de l'usage et de la reconversion du territoire. Le fait passé, l'histoire petite ou grande devient un enjeu pour notre ère de la simulation. La panoplie est recomposée à la perfection et nous attend. Les objets comme les situations sont une représentation sélectionnée d'un fragment du passé mise en service pour nous. Nous pouvons rejouer à volonté, à satiété, une illusion pour nous attacher un morceau du passé. L'image est là, indéfectible, et nous nous rassérénons, nous l'emportons, la diffusons dans l'instant pour que tout le monde sache que nous étions là. Il n'y a pas de falsification, nous avons l'expérience programmée. Le passé est le support d'une simulation précise, froide que nous expérimentons avec un plaisir réitérable à volonté. La scène a été réanimée comme pour le Titanic et se rejoue en boucle. Adolfo Bioy Casares dans L'Invention de Morel (1992) projette une duplication de notre monde. Dans son roman, un homme évadé échoue sur une île déserte et assiste à l'apparition quotidienne d'un monde disparu, peuplé de personnages et de décors aux apparences réelles. Cette société qui revit devant ses yeux est en fait créée grâce à une machine dont l'énergie est inépuisable. Un rêve qui nous garantit l'éternité. La machine à images provoque chez le fugitif une mise en abyme. Elle questionne sur la réalité des images et sur celle que nous souhaitons finalement qu'elles aient.

La tentation de ces nouveaux espaces est une relation ambiguë aux choses et aux événements non vécus puisqu'il s'agit de vivre un moment recomposé et de choisir d'entrer dans une fiction. L'image diffusée de cette fiction deviendra réelle par celui qui va la percevoir. La photographie est là pour que le simulacre soit parfaitement authentique et le fixe dans une nouvelle réalité. 


\section{BIBLIOGRAPHIE}

Appadurai, A., « Definitions: Commodity and Commodification », dans Ertman, M., Williams, J. C., (ed.), Rethinking Commodification: Cases and Readings in Law and Culture, New York, University Press, 2005.

Aristote, Opuscules. Traité de la mémoire et de la réminiscence, traduction par B. Saint-Hilaire, URL : http://remacle.org/bloodwolf/philosophes/Aristote/memoire.htm.

Ballif, F., « Belfast en temps de paix : la guerre civile comme attraction touristique ", Téoros, 34, 1-2, 2015, URL : https://journals.openedition.org/teoros/2761\#quotation.

Barthes, R., Mythologies, Paris, Éditions du Seuil, 1970.

Baudrillard, J., Le Système des objets, Paris, Gallimard, 1968.

Baudrillard, J., « Disneyworld Company », Libération, 4 mars 1996.

Bioy Casares, A., L'Invention de Morel (1973), traduit de l'argentin par Armand Pierhal, Paris, $10 \times 18,1992$.

Borgmann, A., « The nature of reality and the reality of nature », dans Soulé, M. E. et Lease, G. (ed.), Reinventing nature ? Responses to postmodern deconstruction, Washington D.C., Island Press, 1995.

Chevalier, D., « Titanic Belfast vs mémoires d'un naufrage : le patrimoine (post)industriel de la ville ne sombrera pas!», Belgeo, 1, 2014.

Corbin, A., Le Territoire du vide. L'occident et le désir du rivage (1750-1840), Paris, Flammarion, coll. «Champs Histoire », 1990, 407 p.

Debord, G., La Société du spectacle, Paris, Buchet/Chastel, 1967.

Foucault, M., « Des espaces autres. Dits et écrits (conférence au Cercle d'études architecturales, 14 mars 1967), dans Architecture, Mouvement, Continuité, n 5, 1984.

Goeldner-Gianella, L. et Feiss-Jehel, C., D'une rive, l'autre, paysages et scènes des bords de l'eau Peintres anglais et français au XIX ${ }^{e}$ siècle, Pictorial and Social Representations of the Coastline: the Ties that Bind, Mauguio, Pure impression, 2013, p. 62-76.

Gorz, A., Le Traître suivi du vieillissement, Paris, Gallimard, coll « Folio », 2005.

Harvey, D., Géographie et capital. Vers un matérialisme historico-géographique, Paris, Syllepse, 2010.

Hayles, K. N. « Simulated nature and natural simulations: rethinking the relation between the beholder and the world ", dans Cronon, W. (ed.), Uncommon ground. Rethinking the human place in nature, Norton, New York, 1995, p. 411.

Ehrmann, S. « Imaginer ce qui est déjà », « Journée d'étude : paysages et imagination » du Lacth, 2011.

Gunthert, A., «L'image conversationnelle », Études photographiques, n³1, 2014, URL : https:// journals.openedition.org/etudesphotographiques/3387.

Jackson, J. B., De la nécessité des ruines et autres sujets, Fermanville, Éditions du Linteau, 2005.

Jehel, P.-J, Feïss-Jehel, C., « The Photogenic Quality of the Irish Landscape : Reflexions on Photographs of Ireland », LISA, vol. XII, n 3, 2014. 
Kirshenblatt-Gimblett, B. « World Heritage and Cultural Economics », dans Karp, I., Kratz, C. A, Szwaja, L., Ybarra-Frausto, T., (dir.), Frictions de musée, Cultures publiques/Transformations globales, Durham, Duke University Press, 2006, p. 161-202.

Latarjet, B. et Hers, F., Paysages, Photographies, Travaux en cours en 1984-1985, Paris, Hazan, 1985.

Lévi-Strauss, C., Tristes Tropiques, Paris, Plon, 1955.

Lennon, J., Foley, M, Dark Tourism, London, Continuum, 2000, 256 p.

Luginbühl, Y., La Mise en scène du monde. Construction du paysage européen, Paris, CNRS éditions, 2012.

Mannoni, P., Les Représentations sociales, Paris, Puf, 1998, p 25.

Marin, L. « Le maintenant utopique » dans Furter, P. et Raulet, G. (dir.), Stratégies de l'utopie, Paris, Galilée, 1979.

Puech, M., «Don Ihde : la phénoménologie dans la philosophie américaine de la technologie » dans Schmit, P.-É. et Chardel, P.-A. (dir.), Phénoménologie et technique(s), Paris, Éditions du Cercle herméneutique, 2008.

Rea, P., « How useful is it to understand Belfast as a Global City? Divided Cities/Contested States ", Electronic Working Paper Series, n² 26, 2012, URL : http://www.conflictincities.org/ workingpapers.html.

Roger, A., Court Traité du paysage, Paris, Gallimard, 1997.

Wolf, M. J., Entertainment economy. How Mega-Media forces are transforming our lives, New York Random House, 1999.

\section{NOTES}

1. Stéréotype véhiculé par l'image photographique.

2. https://www.wildatlanticway.com.

3. http://www.guide-irlande.com/sites-touristiques/falaises-de-moher/.

4. http://www.terresceltes.net/irlande/burren.

5. https://www.visitbritainshop.com.

6. Voir La Vierge du chancelier Rollin de Jan van Eyck.

7. "Look, touch and listen to the story of Navan and its legend... a chance to experience to being a celt...a memorable and interactive experience" peut-on lire sur la brochure du Navan Center \& Fort (www.navan.com).

8. "Don't just see the Giant's Causeway - experience it. » encourage le National trust d'Irlande du Nord, URL: https://www.nationaltrust.org.uk/giants-causeway/features/your-experience-atgiants-causeway.

9. Voir http://www.causey.ie.

10. Voir https://www.ireland.com.

11. Voir Wolf, M. J., 1999.

12. Voir https://www.guide-irlande.com/sites-touristiques/titanic-belfast-experience.

13. Voir https://www.guide-irlande.com/sites-touristiques/titanic-belfast-experience/.

14. Voir Chevalier, 2014. "Sous une apparence ultramoderne, le bâtiment (le musée), financé essentiellement par les autorités locales et le gouvernement de la province britannique, a coûté près de 97 millions de livres (soit environ 120 millions d'euros). 
15. Ibid. «le Titanic Quarter, financé en partenariat par le conseil touristique de l'Irlande du Nord, le conseil municipal de Belfast, les commissaires du port de Belfast et Titanic Quarter Ltd. » 16. Derry fut choisie en 2013 comme la ville de la culture du Royaume-Uni, devant Birmingham, Sheffield et Norwich.

17. « Murals UK's Top Attraction », BBC News, 6 août 2007.

18. https://www.belfasttours.com/.

19. La commodification correspond à la transformation de quoi que ce soit en marchandise ; « anything intended for exchange ».

\section{RÉSUMÉS}

Les paysages et leurs imageries au sens de l'ensemble des images produites, diffusées ou commercialisées occupent une place privilégiée dans le tourisme irlandais. Nous aborderons ces représentations par un corpus d'images publicitaires et au travers de photographies personnelles. Les «terres de contraste» forment autant de clichés soutenus par les discours et les représentations publicitaires. Le monde ainsi photographié devient un décor où les stéréotypes constituent un imagier (collection d'images) dans lequel nous pourrons nous déplacer lors de notre visite. Des aménagements paysagers construisent en effet aujourd'hui une muséographie des territoires conforme à ces stéréotypes. Nous évoquerons ici la fabrication en Irlande de certains de ces mondes clos, simulacres de la réalité, qui deviennent des objets de consommation visuelle. Les restitutions historiques - les murals de Derry ou de Belfast, les nouveaux quartiers du Titanic, l'histoire populaire - occupent une place privilégiée dans ces choix.

Landscapes and the images of them which are produced, broadcast or marketed, occupy a privileged place in the Irish tourist industry. We will approach these representations through advertising images and personal photographs. "Contrasting Lands" (in French, "Terres de constraste") are pictures accompanied by advertising messages and representations. The world thus photographed presents a series of stereotypes through which we will travel during our visit. Landscaping developments are now creating a museography of territories which corresponds to these stereotypes. In this article we will discuss the invention of some of these closed worlds in Ireland, imitations of reality which are transformed into visual consumer goods. Historical reconstructions - the murals of Derry or Belfast, the new areas within the district of Titanic, and popular history - play a major role in these choices.

\section{INDEX}

Mots-clés : paysage, imagerie, littoral, arts visuels, Irlande

Keywords : landscape, imagery, coastline, visual arts, Ireland 


\section{AUTEURS}

\section{CORINNE FEİSS-JEHEL}

Corinne Feïss-Jehel est maître de conférences en géographie à EPHE-PSL et rattachée à l'UMR LETG 6554. Ses études géographiques croisent différentes sensibilités des sciences sociales et des arts visuels afin de proposer des analyses des paysages et de leurs représentations.

corinne.jehel[at]gmail[dot]com

\section{PIERRE-JÉRÔME JEHEL}

Pierre-Jérôme Jehel est photographe, professeur à l'École de l'image, Gobelins. La question du paysage et de la relation entre l'homme et la nature est un axe important de sa pratique où la photographie apparaît comme un dialogue avec les apparences. Il cherche une dimension mentale : la mémoire, la signification d'un lieu, la mythologie...

jerome.jehel[at]gmail[dot]com 